Bull. Mater. Sci., Vol. 21, No. 3, June 1998, pp. 213-217. (C) Indian Academy of Sciences.

\title{
Electrical properties of zirconium diselenide single crystals grown by iodine transport method
}

\author{
S G PATEL*, M K AGARWAL, N M BATRA ${ }^{\dagger}$ and D LAKSHMINARAYANA ${ }^{\ddagger}$ \\ Department of Physics, 'Department of Materials Science, ${ }^{\ddagger}$ Department of Electronics, Sardar Patel University, \\ Vallabh Vidyanagar 388120 , India \\ MS received 26 August 1997; revised 3 April 1998
}

\begin{abstract}
Single crystals of zirconium diselenide $\left(\mathrm{ZrSe}_{2}\right)$ were grown by chemical vapour transport method using iodine as the transporting agent. The crystals were found to exhibit metallic behaviour in the temperature range 77-300 $\mathrm{K}$ and semiconducting nature in $300-443 \mathrm{~K}$ range. The measurements of thermoelectric power and conductivity enabled the determination of both carrier mobility and carrier concentration. The variation of carrier mobility and carrier concentration with temperature indicates the presence of deep trapping centres and their reduction with temperature in these crystals.
\end{abstract}

Keywords. Zirconium diselenide; chemical vapour transport; electrical conductivity; thermoelectric power; trapping centres.

\section{Introduction}

Zirconium diselenide $\left(\mathrm{ZrSe}_{2}\right)$ crystallizes in a cadmium iodide type structure, which is characterized by strong bonding within layers and relatively weak Van der Waal's bonding between adjacent sheets of layers. The structure is markedly anisotropic giving rise to an extended growth perpendicular to $\mathrm{c}$-axis of the hexagonal crystals. These crystals have interesting electrical (Lee et al 1969a), and semiconducting (Lee et al 1969b) properties. They also exhibit some interesting switching and memory effects (Lee et al 1969a). A possibility of their use in solid state solar cell has also been predicted (Tributsch 1980).

The data concerning electrical properties of $\mathrm{ZrSe}_{2}$ in the literature are very scarce and there have been no recent reports. It has been reported that $\mathrm{ZrSe}_{2}$ grown by granular zirconium has a metallic nature whereas samples prepared from powdered zirconium show a semiconducting behaviour (Onuki et al 1982). They have also shown that in the temperature range $50-300 \mathrm{~K}$ the electrical resistivity of layered $\mathrm{ZrSe}_{2}$ is proportional to $T^{2}$. It has also been reported that samples with higher stoichiometry exhibit semiconducting behaviour while the less stoichiometric compounds are metallic (Deniard et al 1987). The normal semiconducting behaviour of $\mathrm{ZrSe}_{2}$ has also been reported (Zheng et al 1989) in electrical anisotropic studies in the temperature range $100-800 \mathrm{~K}$. Looking at the anomaly in the resistivity behaviour of samples in the low and high temperature regions, we have carried out these measurements in both the ranges

*Author for correspondence of temperatures. Moreover, the fact is $\mathrm{ZrSe}_{2}$ is expected to have highly anisotropic electrical properties. We have also concentrated on this aspect in the resistivity measurements. In this paper, we, therefore, report the growth, characterization, measurements of d.c. resistivity, thermoelectric power, mobility and anisotropy in electrical resistivity in different temperature ranges.

\section{Experimental}

\subsection{Growth of $\mathrm{ZrSe}_{2}$ single crystals}

Synthesis of zirconium diselenide was first reported by Van Arkel (1924). Later, Hahn and Ness (1957, 1959), McTaggari and Wadsley (1958), Greenway and Nitsche (1965), Gleizes and Jeannin (1970), Remmington et al (1972), Lucovsky et al (1973) and Whitehouse et al (1973) have grown this compound by halogen transport methods. However, in the usual vapour growth experiments the quartz ampoule containing the charge is placed in the furnace in such a way that its bottom is directly in contact with the furnace wall while the upper portion is projected inside the furnace tube. This creates an uneven temperature gradient in the vertical direction leading to the introduction of defects into the grown crystals. In order to avoid this temperature gradient, arrangements were made to keep the quartz ampoule (along with the charge) coaxially within the furnace tube in the present investigation. The length of the quartz ampoule was about $250 \mathrm{~mm}$ with an inner diameter of $20 \mathrm{~mm}$. The hot and cold junctions of the furnace were maintained at $1123 \mathrm{~K}$ and $1073 \mathrm{~K}$, respectively. Iodine was used as a transporting agent and large sized single 
crystals of $\mathrm{ZrSe}_{2}$ were obtained over a growth period of $500 \mathrm{~h}$ at the colder end of the ampoule. The crystals could easily be cleaved along the basal (0001) plane. The structural characterization and the compositional analysis were made by $\mathrm{X}$-ray diffraction and energy dispersive analysis of X-rays (EDAX), respectively.

\subsection{Electrical resistivity measurements}

D.C. electrical resistivity measurements were performed on the freshly cleaved (0001) basal planes in a direction perpendicular to c-axis. Low temperature resistivity measurements along the basal planes $\left(\rho_{\| 1}\right)$ in the temperature range $77-300 \mathrm{~K}$ were made in a specially designed cryostat LN-DP manufactured by Scientific Solutions, Mumbai. Electrical contacts were made using proper solder/silver paste. Four point contacts were used to obtain the resistivity. For resistivity $\left(\rho_{\|}\right)$measurements in the temperature range $303-443 \mathrm{~K}$ a four-probe set up manufactured by Scientific Equipments, Roorkee was used. Resistivity measurements normal to the basal plane $\left(\rho_{\perp}\right)$ were carried out on an experimental set up designed and prepared by the University Science and Instrumentation Centre (USIC), Sardar Patel University, Vallabh Vidyanagar. Starting from room temperature $(303 \mathrm{~K})$, the temperature of the sample was increased slowly in steps of $10 \mathrm{~K}$ until a temperature of $443 \mathrm{~K}$ was reached. At each step the corresponding value of resistivity was evaluated.

\subsection{Thermoelectric power measurements}

The thermoelectric power (TEP) measurements were carried out using the thermopower $(S)$ measurements set up TPSS-200 developed by Scientific Solutions, Mumbai. In all the measurements $\Delta T$ was kept as $4 \mathrm{~K}$ and the
TEP $\left(S_{\|}\right)$measurements were carried out in the temperature range 313-443 K. Due to experimental limitations, TEP $\left(S_{\perp}\right)$ measurements normal to the basal plane i.e. parallel to c-axis, could not be made.

\section{Experimental}

\subsection{Structural characterization}

Figure 1 shows the zirconium diselenide $\left(\mathrm{ZrSe}_{2}\right)$ single crystals obtained in the present investigation. Figure 2 shows an a-axis oscillation photograph corresponding to the cleaved front face of $\mathrm{ZrSe}_{2}$ crystals taken with $\mathrm{CuK} \alpha$ radiation employing a conventional $\mathrm{X}$-ray tube with a camera radius of $3 \mathrm{~cm}$ and collimeter aperture of 0.1 $\mathrm{cm}$. The crystal was oscillated about the a-axis between $25-40^{\circ}$. The a-axis oscillation photograph shows rows of constant $h, k$ and varying $l$. The presence of spots along the rows clearly indicates that the grown crystals have an ordered single crystal nature. The values of lattice parameters obtained from the $\mathrm{X}$-ray diffraction are found to be $a=3.772 \AA$ and $c=6.122 \AA$ clearly matching with the reported values (Whitehouse et al 1973) for the compound $\mathrm{ZrSe}_{1.96 \pm 0.3}$. The composition of the crystals as obtained by EDAX correspond to $\mathrm{ZrSe}_{1.96}$.

\subsection{Variation of resistivity with temperature}

Results of low temperature $(77-300 \mathrm{~K})$ resistivity $\left(\rho_{\|}\right)$ measurements on a representative sample are shown in figure 3. It is seen that resistivity increases with increase in temperature and is found to be proportional to $T^{2}$ similar to $\mathrm{TiS}_{2}$ and $\mathrm{ZrSe}_{2}$ as reported by Onuki et al (1982).

In the temperature range $303-443 \mathrm{~K}$ it is seen that

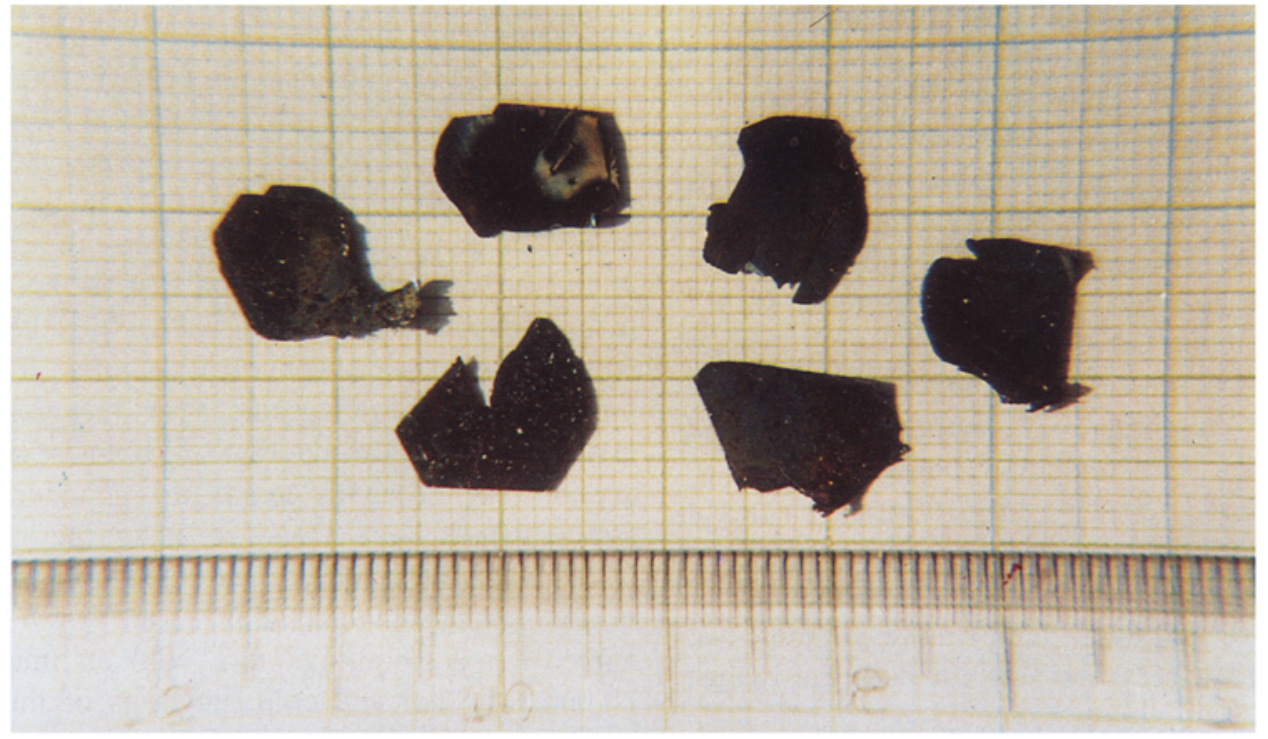

Figure 1. Single crystals of $\mathrm{ZrSe}_{2}$. 


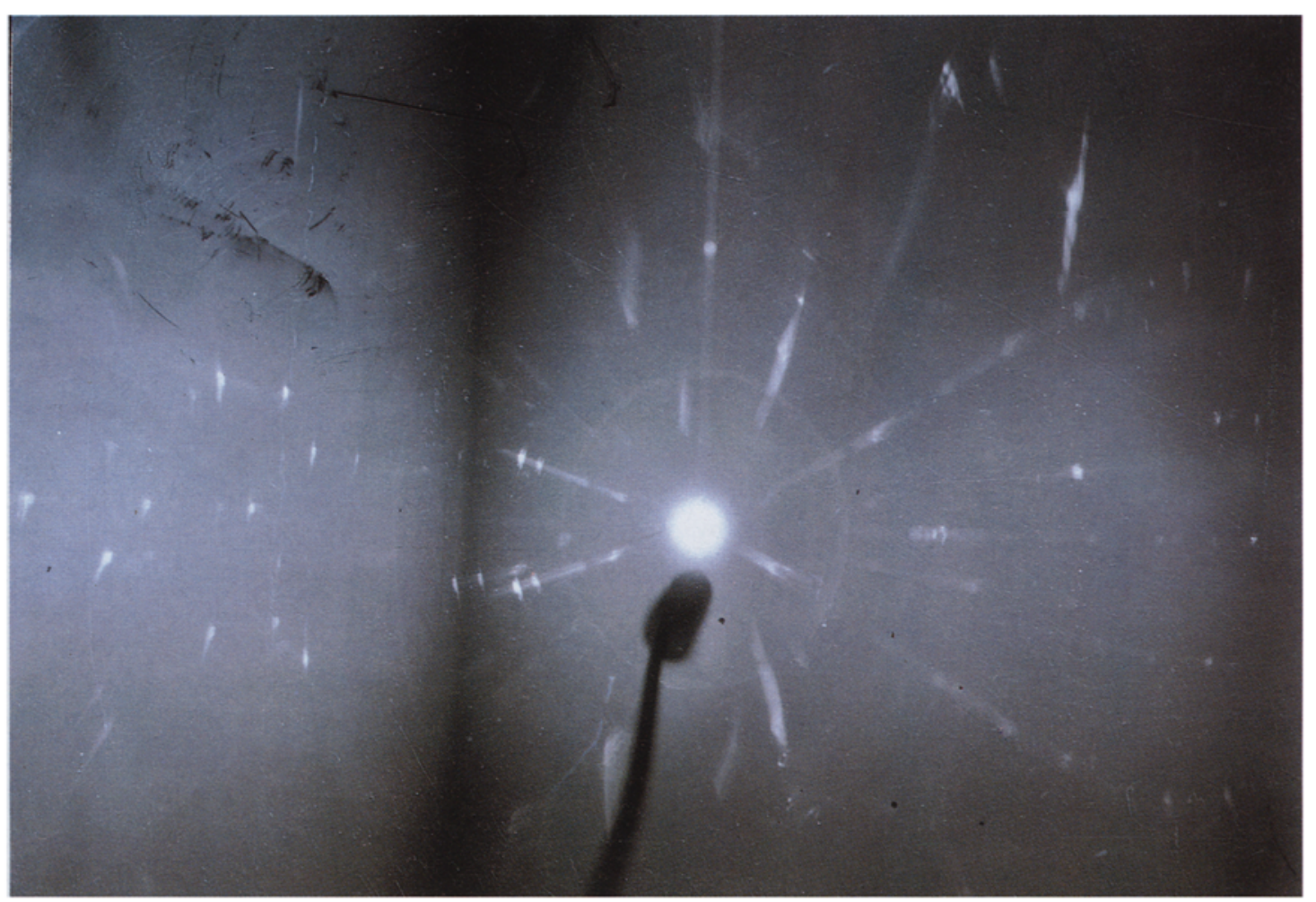

Figure 2. X-ray oscillation photograph of $\mathrm{ZrSe}_{2}$ single crystal.

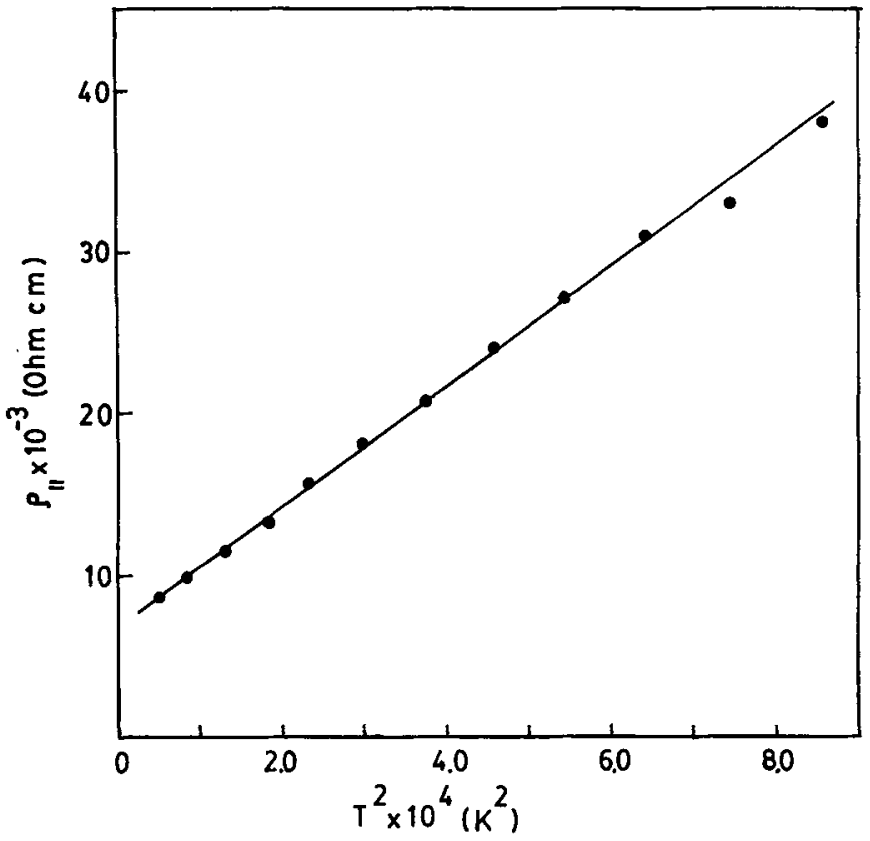

Figure 3. Variation of resistivity with square of temperature.

the resistivity decreases with an increase in temperature showing a semiconducting behaviour. The activation energies obtained from the graph (figure 4) are $0.0587 \mathrm{eV}$ in the temperature range $303-393 \mathrm{~K}$ and $0.187 \mathrm{eV}$ in the temperature range $393-443 \mathrm{~K}$.

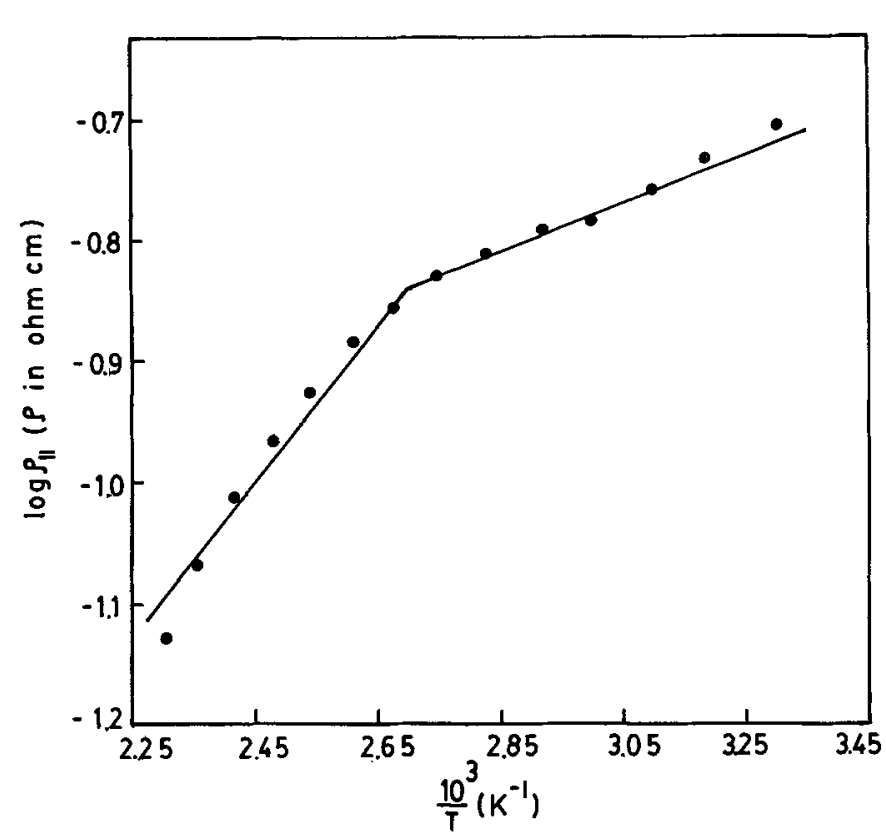

Figure 4. Variation of resistivity with reciprocal of temperature.

3.3 Variation of electrical resistivity anisotropy with temperature

Zheng et al (1989) measured anisotropy in the electrical resistivity $\left(\rho_{\perp} / \rho_{\|}\right)$, in the temperature range $100-300 \mathrm{~K}$. 
In our investigation anisotropy measurements have been carried out in the temperature range $303-443 \mathrm{~K}$. It is seen in figure 5 that the anisotropy $\left(\rho_{1} / \rho_{\|}\right)$ratio increases with increase in temperature. This variation of anisotropy with temperature shows an identical behaviour to that reported by Zheng et al (1989).

\subsection{Variation of TEP with temperature}

The polarity of the thermally generated voltage at the hot end was positive indicating that the crystals are $n$-type in nature. The TEP was found to increase linearly with temperature. The TEP $\left(S=S_{\|}\right)$has been used to evaluate the carrier mobility using the relation given by Lee et al (1969b):

$$
\mathrm{TEP}=S=-\frac{k}{e}\left\{\frac{E_{\mathrm{a}}}{k T}+A\right\},
$$

or

$$
S=-\frac{k}{e}\left\{A+\ln \left[2\left(2 m_{\mathrm{e}}^{*} k T\right)^{3 / 2} / n h^{3}\right]\right\},
$$

where $A$ is the thermoelectric factor, $n$ the electron density, $h$ the Planck constant and $m_{e}^{*}$ the effective mass of electron. The above equation after the substitution of various constants and taking $A=1.5$ for ionized impurity scattering simplifies to

$$
\log n=(3 / 2) \log T-0.005 S+15 \cdot 719 \text {. }
$$

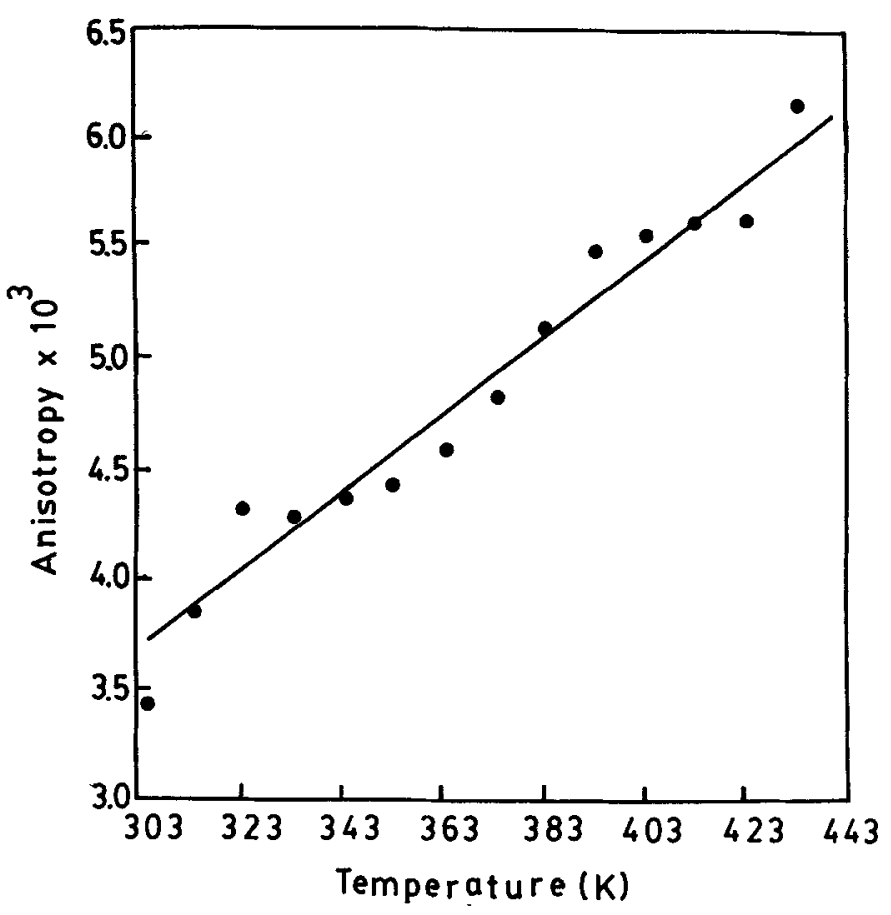

Figure 5. Variation of anisotropy with temperature.
The electron density has been evaluated by using the above relation. The mobility, $\mu$, of the charge carriers is determined from the relation

$$
\mu=\frac{\sigma}{n e},
$$

where $n$ is the carrier concentration, $e$ the charge of electron and $\sigma$ the electrical conductivity.

\section{Discussion}

The $T^{2}$ dependence of electrical resistivity can be attributed to carrier scattering rather than carrier activation. However, due to experimental limitations the scattering and degeneracy could not be studied in detail in this range $(77-300 \mathrm{~K})$ of temperature. The high value of resistivity in the perpendicular direction suggests that conduction of electron in the direction normal to the layer is very little. Further, the anisotropic behaviour and its variation with temperature clearly shows that electronic conduction in $\mathrm{ZrSe}_{2}$ is highly anisotropic. This large anisotropy in electronic behaviour may be attributed to the sufficiently larger effective mass anisotropy $m_{\| 1}^{*}$ being 0.43 whereas $m_{\perp}^{*}$ being 7.00 , where $\|$ and $\perp$ denote, respectively, parallel and perpendicular to the layers of $\mathrm{ZrSe}_{2}$ single crystals (Isomaki et al 1979). The high values of resistivity and large anisotropy further suggest that electron states in $\mathrm{ZrSe}_{2}$ is two dimensional in contrast to a three dimensional behaviour seen in $\mathrm{GaSe}$ and SnSe. The low values of activation energy obtained from figure 4 can be attributed to the high carrier concentration density which gives rise to energy levels very close to the conduction band. These carriers will also be scattered by various centres or regions such as grain boundaries, dislocations, surface states etc in addition to the normally observed scattering modes, viz. ionized impurity, lattice or phonon, piezoelectric etc. The positive temperature dependence of mobility suggests that ionized impurity scattering might be the dominant scattering mechanism in these crystals in the observed temperature range. From the slope of temperature dependent mobility graph (figure 6), it is possible to obtain some qualitative idea about the scattering mode. Since all scattering processes are likely to follow the relation $\mu \propto T^{\mathrm{x}}$, the magnitude and sign of $\mathrm{x}$ will determine the scattering mode. In the present case the value of $x$ from figure 6 comes to about 0.05 and is positive. The theoretical value of $\mathrm{x}$ for ionized impurity acting as a single mode of scattering is $3 / 2$. The deviation in the observed value seems to be due to the superimposition of the ionized impurity scattering with some of the above mentioned scattering processes. The observed range of temperature i.e. $303-443 \mathrm{~K}$, corresponds to the extrinsic/impurity range only as seen from the low values of conductivity activation energy. Also, the carrier concen- 


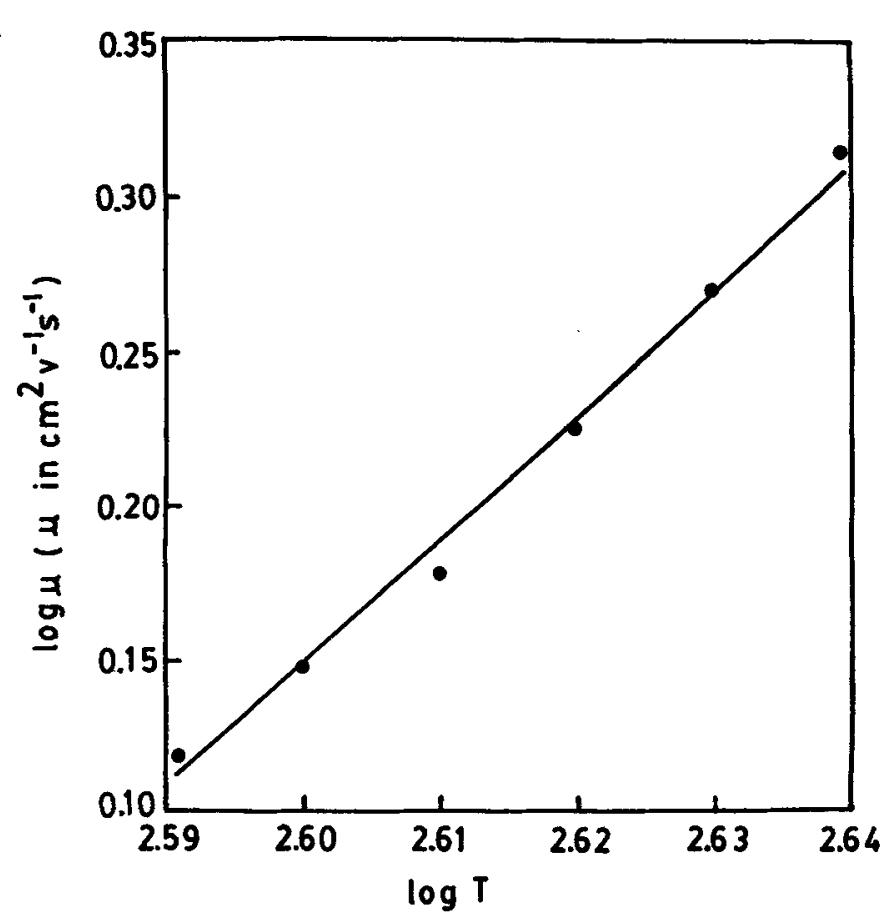

Figure 6. Variation of mobility with temperature.

tration can be expressed as

$$
n \approx \exp \left(\frac{E n}{k T}\right),
$$

where $E n$ is the carrier activation energy of the $n$-type $\mathrm{ZrSe}_{2}$ single crystal. The above equation predicts a linear relation between $\log n$ and $1 / T$ as observed in figure 7 . The carrier activation energy estimated from the slope, was found to be $0.29 \mathrm{eV}$. Since this value is much less than half the reported value of electrical band gap of $1.22 \mathrm{eV}$ (Murray et al 1972), the presence of deep donor like levels is possible in this material. Hence, heating in the lower temperature range reduces the number of deep trapping centres and thereby increasing the number of free carriers and carrier mobility. The mobility may reduce only at still higher temperatures where the scattering due to lattice thermal vibration dominates.

\section{Conclusions}

Single crystals of zirconium diselenide could be grown by chemical vapour transport using iodine as the transporting agent. These crystals exhibited metallic behaviour in the temperature range $77-300 \mathrm{~K}$ and semiconducting nature in the temperature range $300-443 \mathrm{~K}$. The positive temperature dependence of carrier mobility suggested that ionized impurity scattering might be the dominant scattering mechanism in these crystals. The variation of carrier mobility and carrier concentration with temperature

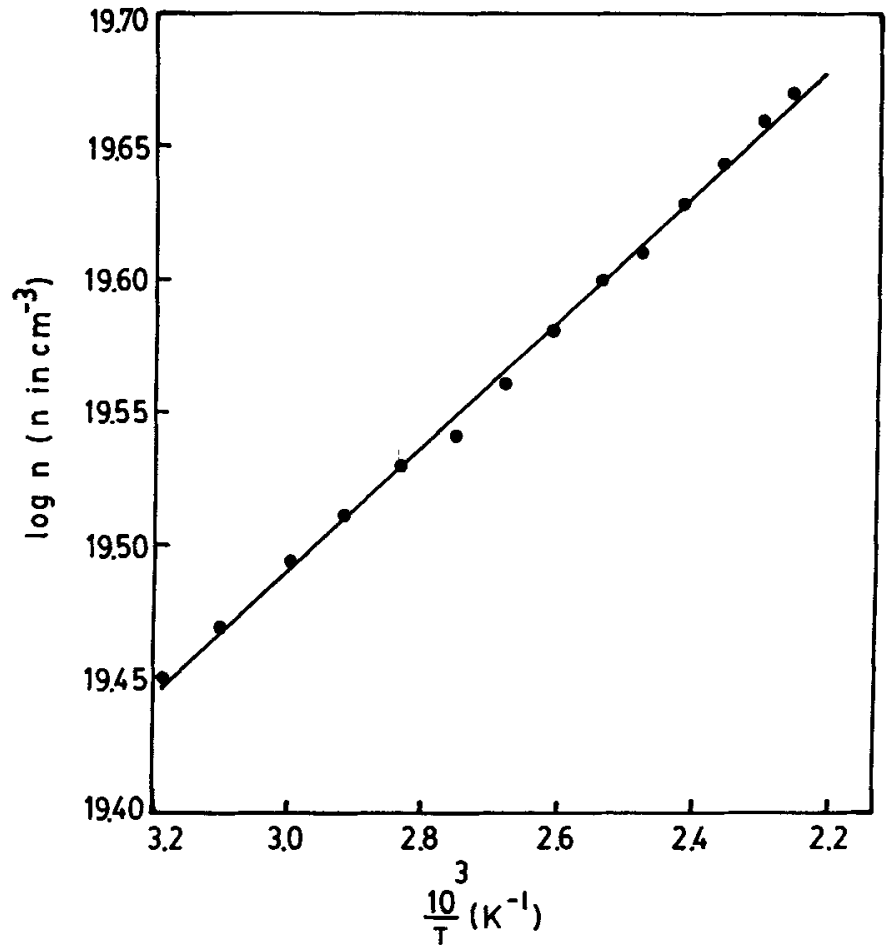

Figure 7. Variation of carrier concentration with reciprocal of temperature.

in the semiconducting range indicates the presence of deep trapping centres.

\section{References}

Deniard P, Chevalier P, Trichet L and Chabre Y 1987 Solid State Commun. 64175

Gleizes A and Jeannin Y 1970 Solid State Chem. 1180

Greenway D L and Nitsche R 1965 J. Phys. Chem. Solids 26 1445

Hahn H and Ness P 1957 Naturwiss. 44534

Hahn H and Ness P 1959 Z. Anorg. Allg. Chem. 30237

Isomaki H, Von Boehm J and Krusivs P 1979 J. Phys. C12 3239

Lee P A, Said G and Devis R 1969a Solid State Commun. 71359

Lee P A, Said G, Devis R and Lim T 1969b J. Phys. Chem. Solids 302719

Lucovsky G, White R M, Benda J A and Revelli J F 1973 Phys. Rev. B7 3859

McTaggari K F and Wadsley A D 1958 Aust. J. Chem. 11445 Murray R B, Bromley R A and Yoffe A D 1972 J. Phys. C5 746

Onuki Y, Inada R and Tanuma S 1982 J. Phys. Soc. Jap. 51 1223

Remmington H P B, Tanner B K and Balchin A A $1972 J$. Cryst. Growth 1551

Tributsch H 1980 Appl. Phys. 2361

Van Arkel A E 1924 Physica 4286

Whitehouse C R, Remmington H P B and Balchin A A 1973 Phys. Status Solidi (a) 18623

Zheng X, Kuriyaki H and Hirakawa K 1989 J. Phys. Soc. Jap. 58622 